\title{
The multi-item localization (MILO) task: Measuring the spatiotemporal context of vision for action
}

\author{
IAN M. THORNTON \\ Max Planck Institute for Biological Cybernetics, Tübingen, Germany \\ and \\ TODD S. HOROWITZ \\ Brigham and Women's Hospital, Boston, Massachusetts \\ and Harvard Medical School, Boston, Massachusetts
}

\begin{abstract}
We describe a new multi-item localization task that can be used to probe the temporal and spatial contexts of search-like behaviors. A sequence of four target letters (e.g., E, F, G, and H) was presented among four distractor letters. Observers located the targets in order. Both retrospective and prospective components of performance were examined. The retrospective component was assessed by having target items either vanish or remain once they had been located. This manipulation had little effect on search performance, suggesting that old target items can be efficiently ignored. The prospective component was assessed by shuffling future target and distractor locations after each response. This manipulation revealed that observers typically plan ahead at least one target into the future. However, even when observers cannot plan ahead, they are still able to ignore old targets. These findings suggest that both "what you did" and "what you intend to do" can influence the localization and selection of targets.
\end{abstract}

This article introduces a new task for exploring the sequential selection of multiple target items during searchlike behavior. This multi-item localization (MILO) task differs in a number of respects from traditional visual search paradigms and, in particular, places a strong emphasis on the temporal, as well as the spatial, aspects of behavior. We will begin by describing the novel features of the MILO task and will discuss some of the theoretical considerations that gave rise to them. Next, we will present three experiments that demonstrate how it can be used for measuring human performance. We will conclude by discussing the ease with which the MILO task can be adapted in order to address a wide range of empirical questions.

The main features of the MILO task are illustrated in Figure 1. Observers are first presented with a trial-specific

The authors contributed equally to this research, and authorship order was determined arbitrarily. Thanks are extended to Richard Abrams, Raymond Klein, Cathleen Moore, Jay Pratt, Kimron Shapiro, Jeremy Wolfe, and an anonymous reviewer for useful comments and suggestions and to George Alvarez, Helga Arsenio, Serena Butcher, Megan Hyle, and Stephan Simon for assistance with data collection. Correspondence may be addressed to either author: T. S. Horowitz, Center for Ophthalmic Research, Brigham and Women's Hospital, 64 Sidney St., Suite 170, Cambridge, MA02139 (e-mail: todd@ search.bwh.harvard.edu)or I. M. Thornton, Max Planck Institute for Biological Cybernetics, Spemannstrasse 38, 72076 Tübingen, Germany (e-mail: ian.thornton @ tuebingen.mpg.de).

Note-This article was accepted by the previous editorial team, headed by Neil Macmillan. alphabetical sequence of target items (e.g., the letters E, F, $\mathrm{G}$, and $\mathrm{H}$ ). This sequence is then distributed within a field of four additional distracting items, two preceding and two following the target sequence (e.g., the letters C, D, I, and J). The observers' task is to locate each target in alphabetical order and to select it, using the mouse. Each trial thus yields $N$ reaction times (RTs), where $N$ refers to the length of the target sequence. The experiments reported below all make use of such four-item alphabetic sequences. However, it is important to point out that the task parameters-that is, sequence type (i.e., alphabetic, numeric, or arbitrary), sequence length, sequence complexity (i.e., interitem relationships), the nature and/or number of distracting items, the ease of physically selecting items (e.g., size or proximity of items), and so on-can easily and systematically be varied. A constant stimulus set has been used throughout this article, since our initial goal is to introduce the basic structure and more general applications of the task.

The MILO task clearly has its roots in the well-known visual search paradigm. ${ }^{1}$ In visual search, observers are asked to report on some property of a designated target item (e.g., location or presence/absence) presented among a variable set of distracting items. Such tasks are typically designed so that each trial isolates a single act of selection in the face of competition from the distracting items, measuring a single response. In this way, we have learned a great deal about the basic building blocks of vision, the overall organization of visual information processing, and in particular, the relationship between vision and attention (e.g., Dun- 


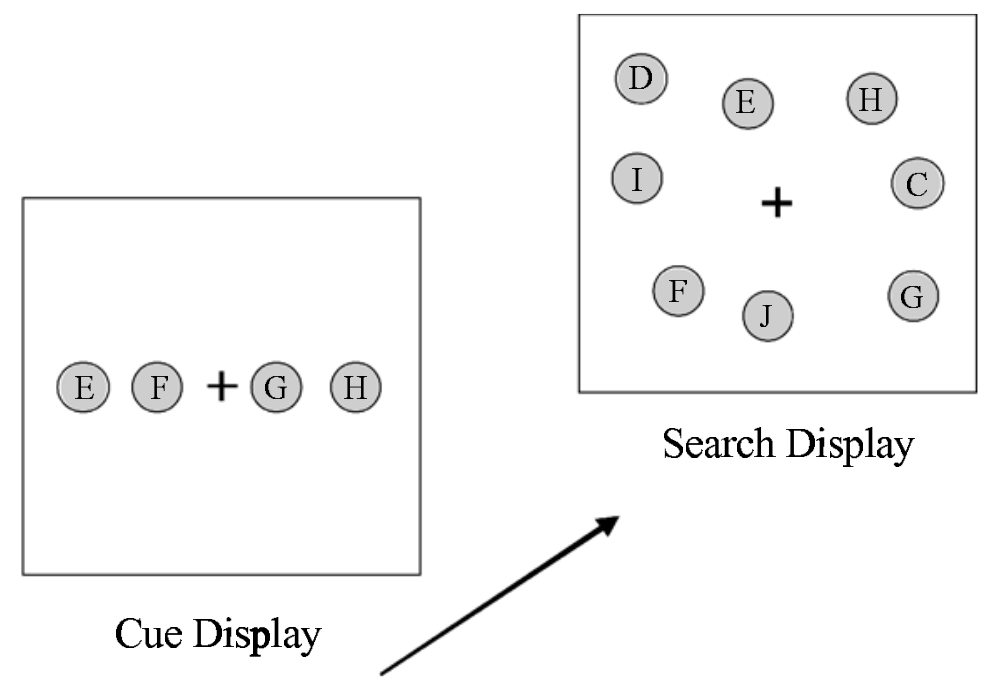

Figure 1. Example stimulus displays.

can \& Humphreys, 1989, 1992; Rensink, \& Enns, 1995; Treisman, 1993; Treisman \& Sato, 1990; Wolfe, 1994, 1998a).

However, in the real world, behaviors rarely occur in isolation. Any given action is likely to be part of a more complex sequence of events. Exploring a sequence of actions is difficult within traditional visual search designs and usually involves indirect measurements, such as the effect of one trial on responses to subsequent trials (e.g., Hillstrom, 2000; Maljkovic \& Nakayama, 1994, 1996; Treisman \& Hayes, 1998). Our goal in designing the MILO task was to provide a more direct way of exploring sequential behavior and, in particular, to place a strong emphasis on the temporal context of that behavior, as well as the spatial context.

More specifically, the studies reported below represent our first attempt to systematically vary and measure the influence of past and future actions on the localization of a current target item. The influence of the past can be manipulated by minimizing the continued influence of targets, by removing them once they had been located, or by maximizing their influence, leaving them visible and unchanged after they had been located (Experiments 1,2, and 3). The future can be manipulated by changing the position of subsequent targets each time the current target is located (Experiments 2 and 3 ). This effectively nullifies any planning that may have taken place.

Another feature of the MILO task is that observers are required to make a direct response-clicking with the mouse - as each target item is found. Again, in the majority of visual search tasks, responses tend to be indirect, with observers asked to report whether or not a target is present, to place it in one of a limited number of perceptual categories, or to fixate it (for an exception, see Meegan \& Tipper, 1999). Although detecting, categorizing, and gazing at objects are clearly important components of behavior, we also need to directly interact with objects-for instance, as we try to navigate around them, reach out and pick them up, or sometimes even run away from them. The use of direct responses in the MILO task is, thus, a shift of focus, as compared with traditional visual search methods, a shift that more closely reflects our everyday interactions with objects in the world (Meegan \& Tipper, 1998, 1999; Tipper, Lortie, \& Baylis, 1992).

The novel features of the MILO task-multiple targets and direct responses - clearly increase the range of phenomena that can be explored, as compared with traditional visual search paradigms. Of course, more complex tasks are also more difficult to interpret. The MILO task includes at least three subcomponents: working memory for encoding and maintaining the target sequence, visual search for locating targets, and the selection and execution of mouse responses. A given pattern of results could conceivably originate with any or all of these component tasks. In the General Discussion section, we will speculate on the most likely basis for the specific patterns of results we observe and will suggest a number of ways in which these subcomponents can be independently manipulated to more directly test their contribution. In the main body of the article, however, we will concentrate on describing the overall behaviorinvolved in locating and responding to multiple target items. In describing this behavior, we will use such terms as locate, respond to, or click on a target in an atheoretical way, encompassing working memory (sequence encoding), perception (search), and action (response).

One of our primary motivations in developing the MILO task was to be able to study the temporal context of search. Several lines of research have recently begun to explore this domain. For example, Rensink and colleagues (Rensink, 2000; Rensink, O'Regan, \& Clark, 2000) have recently described experiments involving search for change, in which information from two displays must be integrated across time. Wolfe and his colleagues (Wolfe, 1999; Wolfe, Klem- 


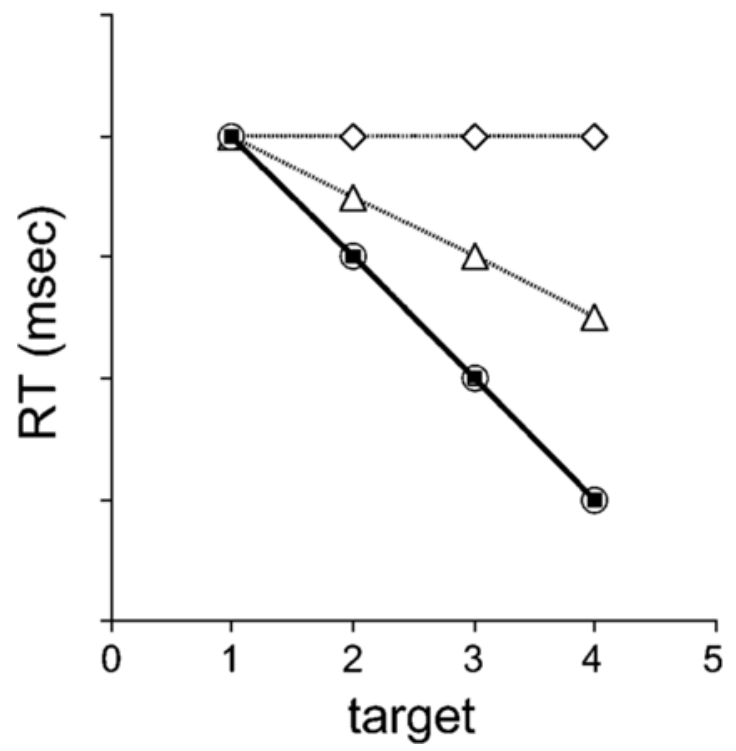

Figure 2. Hypothetical data. Filled squares and solid line indicate the vanishes condition. Open symbols and dotted lines represent various possible outcomes of the remains condition. Circles represent the assumption that prior targets do not influence search. Diamonds represent the assumption that prior targets effectively become distractors. Triangles represent a possible intermediate case.

pen, \& Dahlen, 2000) have investigated the development of the visual representations (or lack thereof) during repeated search through the same display. Watson and his colleagues (Olivers \& Humphreys, 2000; Watson \& Humphreys, 1997, 2000) have introduced a preview paradigm, in which the temporal offset between distractor sets influences the efficiency of search (see also Donk \& Theeuwes, 2001; Gibson \& Jiang, 2001; Theeuwes, Kramer, \& Atchley, 1998). Olson and Chun (2001) have demonstrated that observers can use invariant temporal and spatiotemporal sequences to guide visual attention.

More generally, Shore and Klein (2000) have identified three types of temporal contexteffects in search. In order of decreasing temporal scope, these are long-term perceptual learning (Chun \& Jiang, 1998; Schneider \& Shiffrin, 1977), trial-to-trial priming (Hillstrom, 2000; Maljkovic \& Nakayama, 1994, 1996), and within-trial tracking of attended objects (Klein, 1988; Watson \& Humphreys, 1997). The MILO task is most closely related to the latter category and may shed some light on the current debate in that field.

Next, we will describe the general method of the MILO task in more detail and will present three experiments that probed the temporal context of search behavior.

\section{GENERAL METHOD}

All three of the experiments reported in this article used the same basic method, shown in Figure 1. Each trial began with a cue phase, in which four target items were displayed in the center of the screen. Each target item consisted of a clearly visible oval patch containing a single uppercase letter of the alphabet. The four target letters formed an alphabetic sequence (e.g., E, F, G, and H), which was randomly chosen on each trial. The observers were free to view the target sequence as long as they liked and then initiated the search phase of the trial by pressing the space bar.

The search display contained the four target items plus an additional four distractor items. The distractor items consisted of identical oval patches that each contained one letter from the set of four that immediately preceded and succeeded the target sequence. (e.g., C, D, I, or J). In the search display, the four target and four distractor items were randomly placed within a central viewing area, with the constraint that items never overlapped with each other or with the fixation cross that was shown at the center of the screen. ${ }^{2}$

The observer's task was to locate each of the four target letters in sequence, beginning with the first letter (i.e., E) and ending with the last letter (i.e., H). The location of each target item was identified by clicking anywhere within the target oval, using a standard computer mouse. A trial was terminated and the screen went blank either when the four-item target sequence had been successfully located or when an error had occurred. An error could occur because a mouse click occurred in a nontarget oval, a target item was identified out of sequence, or a mouse click occurred in the background - that is, outside of any of the eight ovals.

The dependent measure of interest was the serial reaction time (SRT), defined as the time to click on target $n$ minus the time to click on target $n-1$. Each trial thus yielded four SRT values. The pattern of these responses, as a function of experimental condition and target position, formed the main focus of analysis in each experiment.

All the experiments were conducted on Power Macintosh computers connected to $75-\mathrm{Hz}$ monitors with a resolution of $1,024 \times$ 768 pixels. Software was custom written, using routines based on work by Steinman and Nawrot (1992), Pelli and Zhang (1991), and Rensink (1990). The observers were seated at a standard viewing distance of $80 \mathrm{~cm}$ in front of the monitor.

\section{EXPERIMENT 1}

The purpose of Experiment 1 was to use the MILO task to explore whether the response to the current target item in a given sequence would be affected by previous responses within the same trial. That is, does "where you've been" affect "where you're going"?

To do this, we compared two basic conditions. In the vanishes condition, target items disappeared from the screen as soon as they were located with the mouse click. This meant that the set size of the display was reduced by one with each subsequent response. Since target items do not pop out in the current displays, identifying them is likely to involve some form of successive inspection. RT thus crucially depends on the total number of items in the display. Within a vanishes trial we would thus predict that responses to the first item, with a set size of eight, should always take longer than responses to the second item, with a set size of seven, and so on, to give an overall RT profile similar to that shown in the filled squares in Figure 2. In the remains condition, target items were completely unaffected by the localizing click of the observer. Thus, the target items remained visible and unchanged after they had been located, and the physical set size remained constant at eight items throughout each trial. The question of interest was how SRT across the four target items would be affected. Some possible outcomes are shown in the open symbols in Figure 2. 


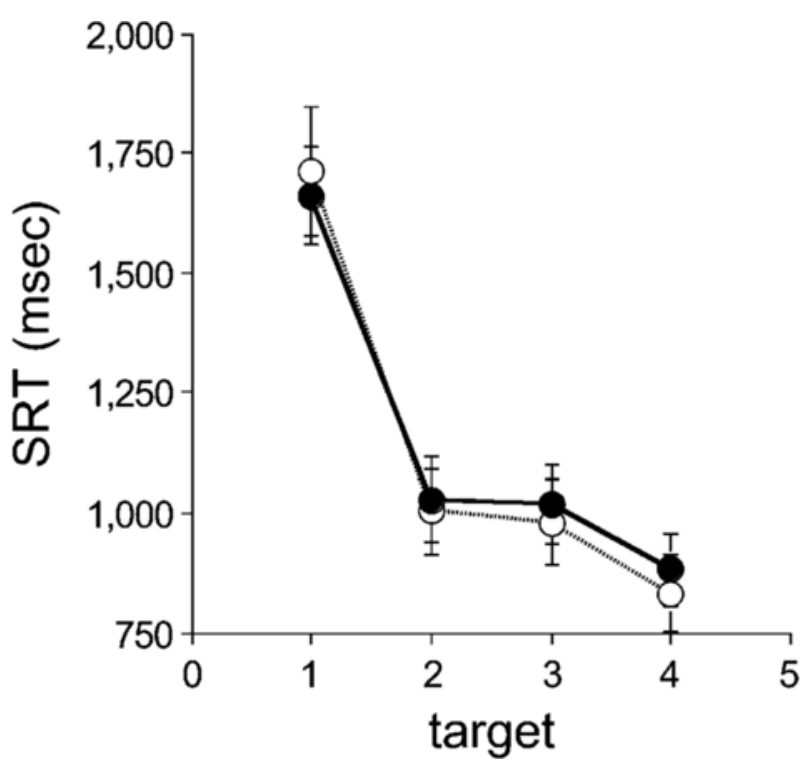

Figure 3. Serial reaction time (SRT) data from Experiment 1. Filled symbols denote the remains condition, and open symbols denote the vanishes condition. Error bars indicate standard errors of the means.

One possibility is that finding one target would not provide the observers with any information that could facilitate search for later targets (e.g., Horowitz \& Wolfe, 1998, 2001, 2003; Wolfe et al., 2000). In this case, SRT should be constant across all targets (diamonds). On the other hand, if observers can exploit memory for the location of previously identified targets, thus eliminating them from subsequent search (Klein \& MacInnes, 1999; Shore \& Klein, 2000; Watson \& Humphreys, 1997, 2000), then we might predict the same pattern of SRT decrease as that seen with the vanishes trials (circles). Another possibility would be for performance to fall midway between these two extremes-for instance, if the observers were able only to mark a subset of the old targets or were not able to completely eliminate them (triangles).

\section{Method}

Subjects. Twelve observers from either the Boston or the Tübingen community were paid for participation in this study. All the observers had normal or corrected-to-normal vision and were naive as to the purpose of the research until after the experimental session had ended.

Stimuli. The stimuli were presented on a uniform, dark gray background that had a central viewing area delineated by a thin black outline square $\left(8^{\circ} \times 8^{\circ}\right.$ of visual angle [DVA]). A central fixation cross $(0.3 \times 0.3$ DVA $)$ was always visible at the center of the screen and was surrounded by a larger, light gray fixation oval $(1.5 \times 1.5 \mathrm{DVA})$. As was described in the General Method section, the cue and target items were small, oval, light gray patches. These patches each subtended approximately 0.5 DVA. The border of the oval was drawn in black, and each item contained a single uppercase letter, also drawn in black. The bounding box of each letter subtended 0.3 DVA.

The target sequence was randomly selected on each trial. The sequence could fall anywhere within the set of alphabetic characters between the letters $\mathrm{C}$ and $\mathrm{X}$. This constraint arose since two distrac- tor items always preceded and followed the target sequence. In the cue display, the four target items were centrally presented, in sequence, with two items either side of fixation. In the search display, the position of all eight items was randomized within the central viewing area on a trial-by-trial basis. The only constraints on position was that the items must be at least 0.5 DVA away from all other items and the central fixation area. In all the experiments, the visible position of a search item was never shifted, although target items were completely removed during the vanishes trials.

Procedure. The observers were first shown several examples of the cue-search trial structure. They were then familiarized with the method of response and were allowed to practice until they were comfortable, using the mouse. The error conditions were illustrated to all the observers, and they were informed that a block would terminate only when they had successfully completed 30 trials. So, although speed of response was emphasized, the observers were also clearly motivated to avoid errors.

The observers completed two blocks of vanishes trials and two blocks of remains trials. Each block contained a minimum of 30 trials. Trials in which an error occurred were replaced, so that a block was finished only when the observers had successfully completed 30 trials. Each observer thus completed a minimum of 120 experimental trials, taking short breaks after each block of $30+$ trials. Block order was counterbalanced, with half of the observers completing the two vanishes blocks before moving on to the remains trials and the other half seeing the opposite order.

\section{Results}

SRTs are shown in Figure 3 for the two conditions as a function of target number. Data were analyzed by a 2 (condition: remains vs. vanishes) $\times 4$ (target ordinal position) repeated measures analysis of variance (ANOVA). There was no effect of condition $\left[F(1,11)<1, M S_{\mathrm{e}}=15,360.78\right]$, nor was there an interaction with target $[F(3,33)=2.077$, $\left.M S_{\mathrm{e}}=5,881.80, p>.1\right]$. However, there was a main effect of target ordinal position $\left[F(3,33)=183.77, M S_{\mathrm{e}}=18,048.59\right.$, $p<.0001]$. A glance at Figure 3 suggests that this effect may be driven primarily by the substantially elevated RTs to the first target. However, when we removed the Target 1 data, the effect was still significant $\left[F(2,22)=34.40, M S_{\mathrm{e}}=\right.$ $5,277.79, p<.0001]$, whereas the main effect of condition and the interaction were not [both $p \mathrm{~s}>.1 ; M S_{\mathrm{e}}$ (condition $)=7,793.49 ; M S_{\mathrm{e}}($ condition $\times$ target $\left.)=2,084.45\right]$. The slope of the SRT $\times$ target function (for the last three targets only) was $79.14 \mathrm{msec} /$ target, which differed significantly from 0 by $t$ test $[t(11)=7.91, p<.00001]$.

Errors were fairly low, averaging $(S E M) 4.2 \%(1.1 \%)$ in the remains condition and $5.7 \%(1.4 \%)$ in the vanishes condition. These two rates did not differ $[F(1,11)=1.51$, $\left.M S_{\mathrm{e}}=0.001, p>.1\right]$.

\section{Discussion}

There were two major findings in Experiment 1. First, the remains and vanishes conditions yielded the same pattern of decreasing SRTs, suggesting that clicking on an item is just as effective in reducing interference as making the item disappear. This clearly implies that during remains trials, old targets could be distinguished in some way from the other items on the screen. Furthermore, this ability extended over time, with the SRT continuing to drop as a function of target. That is, once a target was ignored, it 


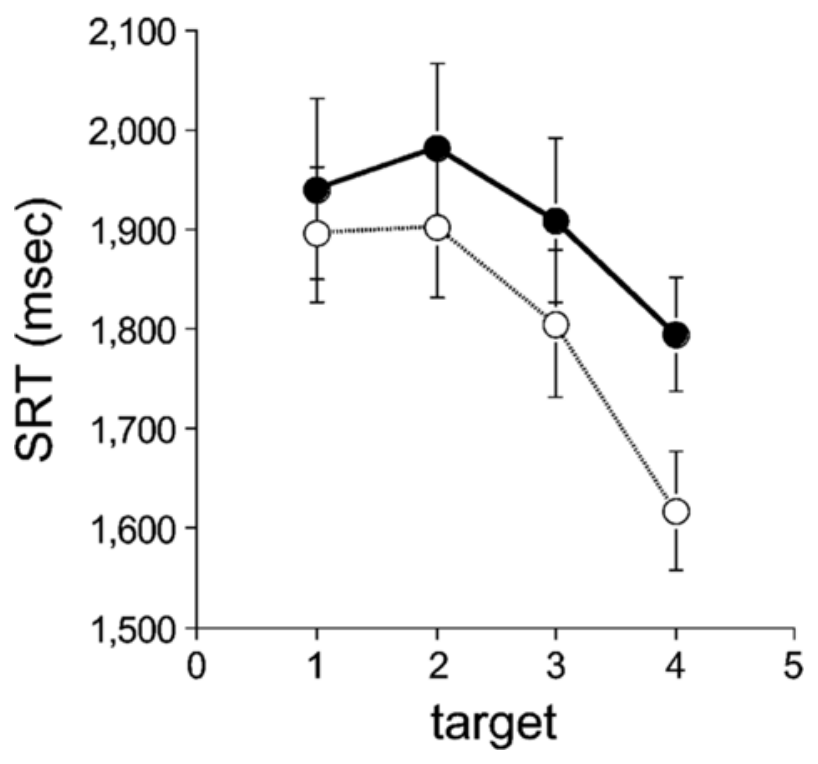

Figure 4. Serial reaction time (SRT) data from Experiment 2. Filled symbols denote the remains condition, and open symbols denote the vanishes condition. Error bars indicate standard errors of the means.

appeared to stay that way for the whole trial. The linear or slightly accelerating SRT profile would not have occurred if previously ignored target locations had begun to regain some influence as the focus of current search moved further away in time.

Second, RTs to the initial target were substantially $(>650 \mathrm{msec})$ slower than SRTs to subsequent targets, which in turn declined by $80 \mathrm{msec} / \mathrm{target}$. There are two possible explanations for this effect. First, the initial latency may simply have been unique. Since the cursor was not visible during the intertrial interval, the subjects had to locate the cursor. There may also have been effects of the initial onset of the stimulus display.

The second explanation is that the subjects may have searched the display and localized all the targets before even planning the first response, in which case SRTs 2-4 simply measured the time taken to move the mouse from one location to the next. Holling worth, Williams, and Henderson (2001) have shown that the identity of previously attended objects can be retained in visual memory during scene viewing. There is also evidence in the sequential movement literature that observers can use visual information to plan movements to at least two targets prior to initiating a response to the first target (Ricker et al., 1999) and that concurrent planning of responses to a second target will slow hand movements to the first target (the firsttarget effect; see Adam et al., 2000). This explanation is loosely compatible with the task chronology observed. The mean RT to the initial target was $1,685 \mathrm{msec}$, which allowed plenty of time for the visual search component of the task to run to completion, assuming a processing time of 50-100 msec/item (Moore, Egeth, Berglan, \& Luck, 1996; Wolfe, 1998a, 1998b). SRTs to subsequent targets were around $1 \mathrm{sec}$, which is somewhat long. Manual tapping responses with more than one target can be executed in less than $200 \mathrm{msec}$ (Adam et al., 2000), single reaching responses in a multiple-target context in less than $500 \mathrm{msec}$ (Keulen, Adam, Fischer, Kuipers, \& Jolles, 2002) and cursor movements in less than $750 \mathrm{msec}$ (Pratt \& Abrams, 1994). It is possible that the two-dimensional cursor movements in our task were executed more slowly than the onedimensional movements in Pratt and Abrams's experiment.

If the observers did search the entire display and localize all the targets prior to initiating the first movement, this may also explain the lack of a difference between the vanish and the remains conditions, since the external stimulus was not being consulted at all in the movement phase. In the next experiment, we tested this idea by requiring the subjects to search after each response.

\section{EXPERIMENT 2}

The goal of Experiment 2 was to test the hypothesis that the observers in Experiment 1 were essentially decomposing the MILO task into separate search and response phases, finding all the targets before executing the first motor response. Our method was to force the observers to follow an iterative search and respond procedure, so that each SRT measured the time to search for that target, as well as any time needed to plan and execute a response. As soon as the observer clicked on a target, the locations of all the items except prior targets were shuffled. If the observers had acquired some information about the location of future targets in the display during their search for the initial target, it would become instantly useless. However, a strategy of tracking prior targets could continue to be useful.

\section{Method}

Subjects. Twelve observers from the Tübingen subject pool were paid for participation in this study. All the observers had normal or corrected-to-normal vision and were naive as to the purpose of the research until after the experimental session had ended. None of the observers had participated in Experiment 1.

Stimuli. The stimuli were identical to those in Experiment 1.

Procedure. The procedure was similar to that in Experiment 1, except that after the observer clicked on a target, the locations of all the distractor letters and subsequent target letters were randomly reshuffled among the current stimulus locations. Targets that had already been clicked on, however, were unchanged. It is important to note that the ovals containing the letters did not change position during a trial. That is, the overall layout of the display remained fixed; only the contents of the ovals (i.e., the letters) were updated. In order to equalize onset and offset transients across all the display items, a global transient was introduced to the display after each target response. When the observers clicked on a target, the center of all the ovals was drawn in with the same gray color as that used for the background. The letters were hidden in this way for $250 \mathrm{msec}$, and then were redisplayed in their shuffled locations.

Each observer then completed a minimum of 120 experimental trials, taking short breaks after each set of 30 trials.

\section{Results}

SRTs for this experiment are shown in Figure 4. The same 2 (condition: remains vs. vanishes) $\times 4$ (target ordinal posi- 
tion) repeated measures ANOVA was used to analyze the data. Overall, the observers were slower in the remains condition by $102 \mathrm{msec}\left[F(1,11)=9.75, M S_{\mathrm{e}}=25,392.31\right.$, $p<.01]$. However, for both, SRTs declined with target $\left[F(3,33)=23.16, M S_{\mathrm{e}}=11,721.92, p<.0001\right]$ at a rate of $72.22 \mathrm{msec} /$ item. This slope was also significantly different from $0[t(11)=7.15, p<.00005]$. There was no interaction between condition and target position $\left[F(3,33)=2.22, M S_{\mathrm{e}}=\right.$ $8,445.20, p>.1]$.

Error rates were again reasonably low, with a mean (SEM) of $5.2 \%(0.9 \%)$ in the remains condition and $6.4 \%$ $(1.3 \%)$ in the vanishes condition. The difference was not significant $\left[F(1,11)=2.31, M S_{\mathrm{e}}=0.04, p>.1\right]$.

\section{Discussion}

Experiment 2 eliminated the large difference between the initial SRT and the remaining three responses. This is consistent with the hypothesis that the observers in Experiment 1 had been locating all of the targets prior to initiating their response to the first target. ${ }^{3}$ On the other hand, the hypothesis that the first latency is somehow unique is disproven. The shuffle manipulation of Experiment 2 forced the observers to use an iterative search and response strategy, eliminating the advantage for later targets. Together, the results of Experiments 1 and 2 suggest that, when it is available, observers can store and use information relating to goals beyond the current target. In the General Discussion section, we will return to this issue and propose some alternative forms that this prospective information might take.

It is also clear from Figure 4 that, even in the absence of useful prospective information, observers are still able to use information about targets to which they have already responded. SRTs declined after the second target in both the vanishes and the remains conditions, indicating that the observers were still able to distinguish prior targets from other display items. This shows that the availability of reliable prospective information is not a prerequisite for utilizing retrospective information.

In contrast to the results of Experiment 1, SRTs were slower overall in the remains condition than in the vanishes condition. This may indicate that some effort was required to suppress prior targets (Watson \& Humphreys, 1997) or that some sort of generalized masking effect occurred as a result of the greater visual clutter in the remains condition.

Interestingly, the gap between the remains and the vanishes conditions grew from $45 \mathrm{msec}$ for the initial target to $177 \mathrm{msec}$ for the fourth target, although the condition $\times$ target position interaction was not statistically significant. This might indicate that the suppression of old targets in the remains condition was imperfect, or leaky, as compared with that in the vanishes condition.

\section{EXPERIMENT 3}

For the final experiment in this series, we wanted to exploit the flexibility of the multitarget paradigm to investigate how far ahead observers can plan. In Experiment 2, we had completely blocked planning by shuffling all of the items ahead of the current target. Here, we used the shuffle manipulation to create four levels of target stability: none (all future targets were shuffled), one (the very next target was left in place, and all the subsequent targets were shuffled), two (the next two targets were left in place), and all (no targets were shuffled, only distractors).

\section{Method}

Subjects. Twelve observers from the Boston population were paid for their time and were naive as to the purpose of the experiment until the session was complete. None of the observers had participated in Experiment 1 or 2.

Procedure. The procedure was similar to that in Experiment 2, with two exceptions. First, we varied (in separate blocks) how far in advance future targets were shuffled. For example, consider a trial on which the target sequence was E, F, G, and H. In the no stable targets condition, when the observer clicked on $\mathrm{E}$, the locations of F, G, and $\mathrm{H}$, along with the four distractors, would be shuffled. This was identical to the procedure in Experiment 2. In the one stable target condition, the $\mathrm{F}$ would remain in the same location, but $\mathrm{G}$ and $\mathrm{H}$ would be shuffled, and so on. Since there were only four targets, the three stable targets condition meant that no targets were shuffled, so we termed this all targets stable. Target stability was varied in blocks. The order of blocks was counterbalanced across subjects. Each block consisted of two sessions of 30 trials each, with a brief break in between.

The second modification relative to Experiment 2 was that the remains versus vanishes manipulation was now carried out between subjects. This was done to reduce the demands on observers, since the number of shuffle conditions would have resulted in a large number of trials.

\section{Results}

SRT data (shown in Figure 5) were submitted to a 2 (group: remains vs. vanishes) $\times 4$ (target) $\times 4$ (target stability: none, one, two, or all) mixed ANOVA, with remains versus vanishes as a between-subjects variable. SRTs did not differ between the two groups $\left[F(1,10)<1, M S_{\mathrm{e}}=\right.$ $709,624.22]$, nor did group interact with any of the other factors (all $F_{\mathrm{S}}<1$ ), indicating that the subjects in the remains condition produced the same pattern of results as the subjects in the vanishes condition. We observed significant main effects of target $\left[F(3,30)=75.69, M S_{\mathrm{e}}=\right.$ $32,307.95, p<.0001]$ and stability $\left[F(3,30)=83.33, M S_{\mathrm{e}}=\right.$ $19,503.16, p<.0001]$, as well as a stability $\times$ target interaction $\left[F(9,90)=25.43, M S_{\mathrm{e}}=7,758.97, p<.0001\right]$. A glance at Figure 5 indicates the source of the interaction: Whereas all the stability conditions appeared to produce similar RTs to the initial target, SRTs to subsequent targets dropped substantially (i.e., 300-500 msec) in conditions with one or more stable targets. This drop was very much shallower (only $1 \mathrm{msec}$ in the remains condition)for the no stable targets condition.

In order to examine this pattern further, we analyzed the data separately for the initial target and Targets $2-4$. The ANOVA on the initial target data showed no significant effects, confirming the impression given by Figure 5. However, the ANOVA of SRTs to subsequent targets showed main effects of target $\left[F(2,20)=35.47, M S_{\mathrm{e}}=7,810.79\right.$, $p<.0001]$ and stability $\left[F(3,30)=105.87, M S_{\mathrm{e}}=\right.$ $20,704.79, p<.0001]$, as well as a stability $\times$ target inter- 


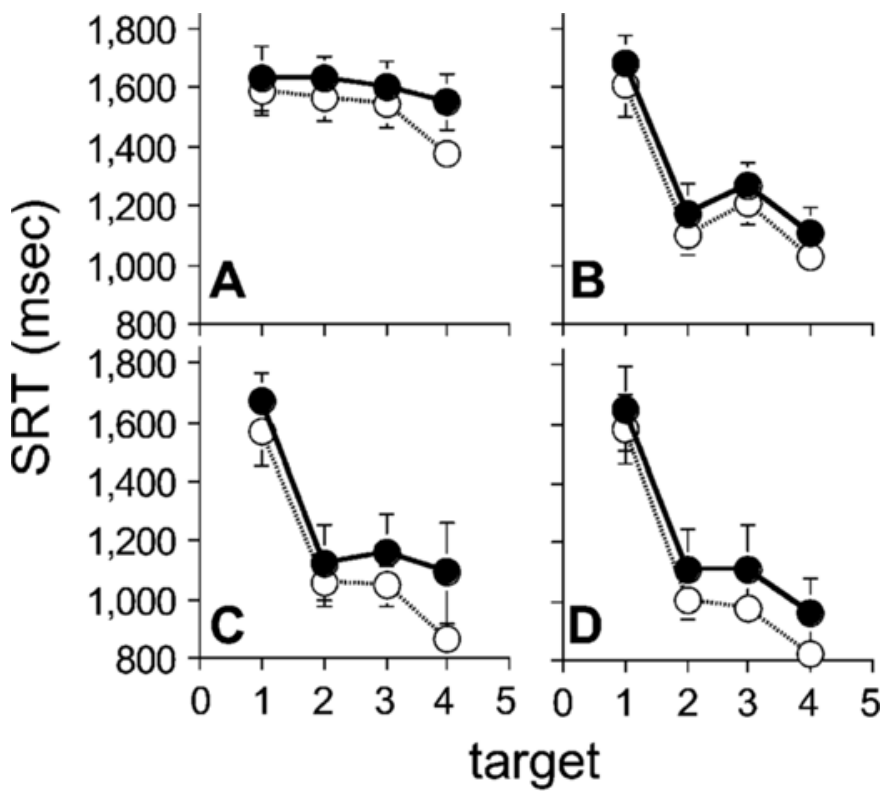

Figure 5. Serial reaction time (SRT) data from Experiment 3. In panels A-D, filled symbols denote the remains condition, and open symbols denote the vanishes condition. Panel A shows data from the no stable targets condition, panel $B$ the one stable target condition, panel $C$ the two stable targets condition, and panel $D$ the three stable targets (or no shuffle) condition. Error bars indicate standard errors of the means.

action $\left[F(6,60)=2.29, M S_{\mathrm{e}}=4,886.76, p<.05\right]$. The target $\times$ group interaction was marginal $[F(2,20)=2.69, p=$ .09]. Although SRT overall declined with target, in fact this was due to the fast SRTs to the fourth target. SRTs to the second and the third targets did not differ (Fisher's PLSD, $p>.10$ ). The effect of the stability factor was likewise due primarily to the large drop from no stable targets to the remaining conditions. However, there was also a significant advantage when the observers could count on the next two targets being stable. Fisher's PLSD showed significant differences between no stable targets and one stable target $(p<.0001)$, one stable target and two stable targets $(p<$ $.05)$, but not between two stable targets and all targets stable $(p=.10)$.

Error rates, shown in Table 1, were again fairly low. An ANOVA of errors, with shuffle and group as factors, revealed no effects.

\section{Discussion}

How far ahead do observers plan in this task? The pattern of RTs was clearly influenced by target stability in a nonlinear fashion. The dramatic decline in SRTs from no stable targets to one stable target tells us that observers are capable of planning one step ahead if the environment will support it. Additional levels of target stability provided smaller advantages. The observers seem to have been taking advantage of the availability of two stable targets on at least some trials. Although there appears to have been a trend of declining SRTs in the data, the advantage for having all three future targets stable did not reach significance.
Additional support for the notion that observers planned one step ahead comes from a peculiar nonlinearity in the effect of target order. Responses to the second target seem to have been as fast (or faster) than responses to the third target. Responses to the second target were also the least variable across all the conditions. These patterns could have arisen because this target was available for planning far longer than any other item. If we assume that, on most trials, the subjects planned one step ahead, the second target was available in a stable location as the next target for (on average) $2,842 \mathrm{msec}$ (SRT to Target $1+$ SRT to Target 2). After the response to the initial target, the third target became the next target and, therefore, the object of planning, for 2,461 msec, and the last target enjoyed this status for $2,340 \mathrm{msec}$. The extra $180 \mathrm{msec}$ may have sped responses to the second target, relative to the other targets.

Did the observers adopt different search strategies as we varied the stability of targets in the display? The fact that RTs to the initial target were unaffected by condition sug-

Table 1

Error Percentages in Experiment 3

\begin{tabular}{cccccc}
\hline & \multicolumn{4}{c}{ Condition } \\
\cline { 2 - 3 } Target & \multicolumn{2}{c}{ Remains } & & \multicolumn{2}{c}{ Vanishes } \\
\cline { 2 - 3 } \cline { 5 - 6 } Number & $M$ & $S E M$ & & $M$ & $S E M$ \\
\hline No stable targets & 3.90 & 1.40 & & 5.96 & 2.98 \\
One stable target & 2.37 & 1.38 & & 3.14 & 1.65 \\
Two stable targets & 3.10 & 1.53 & & 2.42 & 0.81 \\
All targets stable & 2.39 & 0.97 & & 4.70 & 1.38 \\
\hline
\end{tabular}




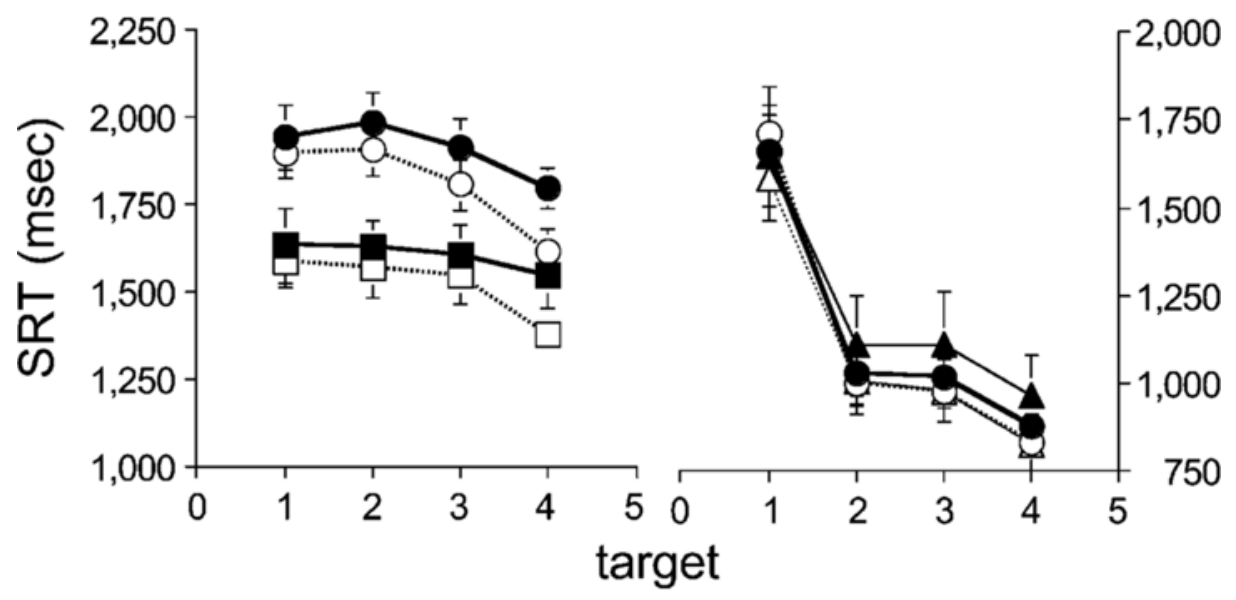

Figure 6. Comparison of the serial reaction time (SRT) data across experiments. Filled symbols denote the remains condition, and open symbols denote the vanishes condition. In the left-hand panel, circles indicate data from Experiment 2, and squares indicate data from the no stable targets condition of Experiment 3. In the right-hand panel, circles indicate data from Experiment 1, and triangles indicate data from the three stable targets condition of Experiment 3. Error bars indicate standard errors of the means.

gests that the observers probably were not using different strategies in different blocks of trials. We can also compare across experiments to gain some insight into this question.

The no stable targets condition in Experiment 3 is a replication of Experiment 2. For comparison, these data are plotted together in the left-hand panel of Figure 6 . The subjects in Experiment 3 were noticeably faster in their responses, and the decline in SRTs from the second to the fourth targets was somewhat shallower. Otherwise, the curves are fairly similar. Specifically, there was little gain from the first to the second targets, followed by an acceleration in the SRTs across the remaining positions. Across both experiments, responses were uniformly slower in the remains condition.

The generally slower SRTs in Experiment 2 could reflect the fact that these observers never experienced stable conditions. Their entire experience of the task was with shuffling of all the items ahead of the current target. To minimize errors, they may have adopted a relatively slow pace. In contrast, the observers in Experiment 3 would also have experienced more stable displays in which faster responses would have been possible. Consistent with this idea, a pilot study for Experiment 2, using observers who had previously seen stable displays, produced a qualitatively similar pattern of results, except that overall SRT was reduced by some $500 \mathrm{msec}$.

The right panel of Figure 6 shows a comparison of Experiment 1 with the all stable targets condition of Experiment 3. Remember that in Experiment 3, unlike in Experiment 1 , each time the observer clicked on a target, there was a global transient, and all of the distractors were shuffled. Nevertheless, the data are highly similar. This tells us two things. First, the shuffling of distractors had little impact on this task, in agreement with the conclusions of Horowitz and Wolfe $(1998,2003)$. Second, global transients had little effect on performance when stable information about future targets was available.

Finally, there was one striking noneffect in Experiment 3: There was no difference between the two groups. The observers produced the same pattern of behavior whether or not we removed old targets from the display.

\section{GENERAL DISCUSSION}

The purpose of these experiments was to allow us to explore both spatial and temporal aspects of an observer's interaction with the environment. Our data suggest that during the course of a trial, observers retain information about targets to which they have already responded. Furthermore, observers also acquire some information about future targets. Thus, observers appear to be able to use both prospective and retrospective memory to facilitate responses within the space of a single trial.

\section{Retrospective Effects}

In introducing Experiment 1, we outlined three hypotheses and the pattern of results they would predict. The data most closely resemble the circles in Figure 2. SRTs in the remains condition generally follow the same course as those in the vanishes condition, except for an additive slowing in Experiment 2, which is not replicated in Experiment 3 . How can we account for this overall pattern of results?

As we noted in the introduction, performance in the MILO task can be decomposed into three main components: (1) memorization of the target sequence, (2) searching for targets, and (3) responding to targets. Since the memory component was not systematically varied in the present experiments and the memory load was so light — the alphabet sequence was presumably overlearned for all of our sub- 
jects - it is unlikely to have had a major impact on performance. In addition, we can note that working memory load has been shown to have little effect on visual search tasks (but see Oh \& Kim, 2002; Ojanpää \& Näsänen, 2002; Woodman, Vogel, \& Luck, 2001). Therefore, in the remainder of this discussion, we will concentrate on the two other components, search and response.

In terms of the search component, the observed pattern of results could be explained by assuming that after a target has been located, subsequent searches through the display ignore that location. Thus, the effective set size decreases with each target. Note that here we are taking a largely atheoretical view of the search component. There are a number of competing theories of visual search, including twostage models (Treisman \& Sato, 1990; Wolfe, 1994), dynamic competition models (Duncan \& Humphreys, 1989, 1992), and signal detection models (Eckstein, Thomas, Palmer, \& Shimozaki, 2000; McElree \& Carrasco, 1999; Palmer \& McLean, 1995; Zenger \& Fahle, 1997). On any of these accounts, there should be a steep set size cost in this task, which accounts for the decreasing SRT with decreases in number of targets.

A number of mechanisms have been proposed to allow the visual system to ignore certain locations. Recent work by Watson and Humphreys (Watson, 2001; Watson \& Humphreys, 1997, 2000) has demonstrated that when observers are given a preview of a subset of locations that will be irrelevant in a subsequent search trial, they can effectively ignore these locations and restrict their search to new items. They have proposed a visual marking mechanism by which irrelevant locations can be suppressed (see also Gibson \& Jiang, 2001; Theeuwes et al., 1998). However, it is unlikely that visual marking is involved here. Visual marking requires at least $400 \mathrm{msec}$ to implement and is subject to interference from competing tasks (Olivers \& Humphreys, 2000). Furthermore, the preview effect does not occur when observers search the preview displays (Olivers, Humphreys, Heinke, \& Cooper, 2002).

More promising is the inhibitory tagging mechanism proposed by Klein (1988). Klein has argued that inhibition of return (IOR; the reduced ability to respond to targets at previously attended locations or objects; see Klein \& Taylor, 1994; McAuliffe, Pratt, \& O’Donnell, 2001; Posner \& Cohen, 1984; Posner, Rafal, Choate, \& Vaughan, 1985; Ro \& Rafal, 1999; Tipper, Jordan, \& Weaver, 1999; Tipper, Weaver, Jerreat, \& Burak, 1994) marks off the locations of rejected distractors during visual search, serving to bias both covert attention (Klein, 1988; Müller \& von Mühlenen, 2000; Takeda \& Yagi, 2000) and overt eye movements (Klein \& MacInnes, 1999) to new locations. Horowitz and Wolfe (1998) have recently challenged whether rejected distractors are, in fact, marked off, and the question remains controversial (Horowitz \& Wolfe, 2001, 2003; Kristjánsson, 2000), especially with regard to eye movements (Gilchrist \& Harvey, 2000; Gilchrist, North, \& Hood, 2001; Peterson, Kramer, Wang, Irwin, \& McCarley, 2001). However, the MILO task differs in important respects from standard visual search tasks. In particular, here we are measuring the putative effects of tagging target locations, which is not inconsistent with Horowitz and Wolfe's account. There is evidence that IOR can be applied to more than four locations in the same display (Snyder \& Kingstone, 2000; Wright \& Richard, 1996), indicating a capacity sufficient for our stimuli. Furthermore, IOR has been demonstrated in experiments in which mouse responses have been used (Briand, Larrison, \& Sereno, 2000; Tanaka \& Shimojo, 1996) and even in some reaching tasks (Fischer, Pratt, \& Neggers, 2003; Howard, Lupiáñez, \& Tipper, 1999).

A third possibility is that observers deliberately construct a representation or layout map of the relevant display and restrict their search to the relevant areas on this map. We know that this is possible in a trivial fashion: $\mathrm{Ob}-$ servers are able to confine their search behavior to the computer monitor and not search the whole room. In a more technical sense, we know that observers can reduce the effective set size of a display when cued in advance (Palmer, 1995). In the MILO task, such a layout map would need to be updated on line after each response as old targets become irrelevant for future behavior.

Recent studies have suggested that observers in search tasks can maintain some memory for the locations of multiple targets during a given trial (Gibson, Li, Skow, Salvagni, \& Cooke, 2000; Horowitz \& Wolfe, 2001). However, in these studies, it was not clear whether such memory merely allowed subjects to determine whether they were examining a target they had looked at before or whether they were able to avoid deploying attention to old targets altogether. The parallel decline in SRT in the remains and vanishes conditions of the present experiments suggests that previously visited targets may be excluded from future searches of the same scene. The hint of a shallower decline (from the second to the fourth targets) in the remains condition in Experiment 2 may indicate that this memory is imperfect.

The idea that a layout map might aid performance seems to be contradicted by the repeated search experiments of Wolfe et al. (2000). Inverting the standard search paradigm, in which observers are asked to report the presence or absence of a constant target in a series of stimulus arrays that change from trial to trial, Wolfe et al. varied the target from trial to trial but left the same stimulus array on the screen for up to 350 trials in a row. They found that observers continued to search anew on each trial, as measured by the persistence of an RT $\times$ set size interaction, despite the fact that they could simply have learned the search array and responded from memory. Wolfe et al. argued that although a memory representation of the search array might well be formed, the cost of checking the visual stimulus, relative to memory search, might remain low enough to discourage the use of a memory-based strategy. That is, if direct visual search of an array is rapid and efficient, the advantages of switching to a memory-based strategy (estimated at $100 \mathrm{msec}$ in their Experiment 6) might not be salient enough for observers to change their strategy. Some support for this explanation comes from another condition 
in that experiment, in which visual transients increased the time taken to search the display, so that memory search was $300-400 \mathrm{msec}$ faster. In this case, there was evidence that the observers began to shift to memory search The added complexity of the MILO task-where observers must make multiple, spatially localized responses to targets that are already held in memory - might substantially change this cost/benefit ratio and make the use of a memory-based layout map much more likely.

Having considered how the search component of the MILO task may have determined performance, we now turn to consider how response selection effects also could have contributed. In their studies of reaching tasks, Tipper et al. (1992) suggested that motor programs to both targets and distractors might be prepared in parallel and that such programs could compete with each other, causing response interference, a finding replicated by Pratt and Abrams (1994) with a cursor movement task rather than with a reaching task (see also Keulen et al., 2002; Meegan \& Tipper, 1998). In these studies, there are typically one target item and one or two distractor items. The MILO task is somewhat more complicated, since there are eight items evoking motor programs, four of which will eventually be released. Whereas Meegan and Tipper $(1998,1999)$ observed interference only in an action-centered frame of reference (i.e., only distractors in between the initial hand location and the target mattered), Keulen et al. (2002) demonstrated that interference would occur in an environment-centered frame of reference (i.e., distractors that were not in the movement path were equally detrimental) when stimuli were closer together, similar to our displays.

We can explain the speedup in SRT as a function of target position by assuming that unexecuted responses compete with each other. Once a response has been made, there is suddenly one less action competing for resources. Thus, after each response, there will be less competition for the next response program to overcome. The reduction in competition as responses are executed would not have been observed in the various reaching experiments, since they required a single response.

Evidence for the argument that response competition could be involved in the pattern of SRTs comes from the appearance of nonlinear declines with target position, particularly in Experiment 2 and the shuffle $n+1$ condition of Experiment 3. Here, although the data showed that responses to the final targets were faster than responses to the initial targets, the functions were distinctly nonlinear. This nonlinearity was present for both the vanish and the remains conditions. Visual search for digits among other digits is known to be reliably linear (Krueger, 1984), especially under varied-mapping conditions, when targets on one trial could be distractors on the next (Schneider \& Shiffrin, 1977). If the reduction in SRT were determined purely by the search component - that is, by the reduction in effective set size - the trend should always be linear.

Could the appearance of nonlinear declines originate at the response level? Studies of reaching have typically measured interference from a single distractor (Meegan \& Tip- per, 1998, 1999; Pratt \& Abrams, 1994; Tipper, Meegan, \& Howard, 2002), so the shape of the interference function is not known. However, the effects of the number of response alternatives are nonlinear (Van Selst \& Jolicœur, 1997).

At least one possible model of response competition could produce the nonlinearity observed in our data. Assume that unexecuted responses compete for a common pool of activation $(A)$ and that all responses $(n)$ begin with roughly equal baseline activation (e.g., $A / n)$. The response to the current target continues to gain activation at the expense of nonmatching responses until some fixed threshold is reached. At this point, the response is executed and removed from the pool. When the weights are reset for the next round of competition, baseline levels of activation $[A /(n-$ 1)] will be shifted closer to the execution threshold - since there is now one less response- thus producing nonlinearly decelerating RT $\times$ target functions.

Finally, it is important to note that search- and responsebased explanations are not mutually exclusive. It is quite possible that we are observing a combination of search-and response-related factors, a possibility that we hope to address with future studies in which the MILO task will be used.

\section{Prospective Effects}

When observers are allowed to plan ahead (Experiment 1), performance is superior to when they are prevented from planning ahead (Experiment 2). Furthermore, this phenomenon is graded (Experiment 3): the further in advance they are allowed to plan, the better their performance, with diminishing returns as information is available further forward in time. This implies that while searching for one target, observers are incidentally picking up information about targets later in the sequence. This does not seem to be a strategic effect, in that search for the initial target takes about the same amount of time whether or not observers expect information about later targets to be useful.

What could be the basis for these prospective effects? Again, we can explain these effects on the basis of either the search component or the response component. We have already suggested that observers develop an internal map of the spatial layout of the display, which could serve to support faster search and/or make it easier to prepare responses. Another possibility is a prospective action-centered representation. For instance, the motor planning to move the cursor to the next target may be occurring while the response to the current target is being planned or executed (Adam et al., 2000; Fischer, Rosenbaum, \& Vaughan, 1997). According to the response competition account developed in the previous section, the prospective representation may take the form of priming the responses to future targets, increasing their activation over that of distractor responses.

Finally, another interesting feature of the data is the gap between the no stable targets condition and the other stability conditions (see Figure 7). Most of the prospective advantage in Experiment 3 comes from the difference between no stable target, where planning is useless, and one stable target, where planning at least one step ahead is pos- 


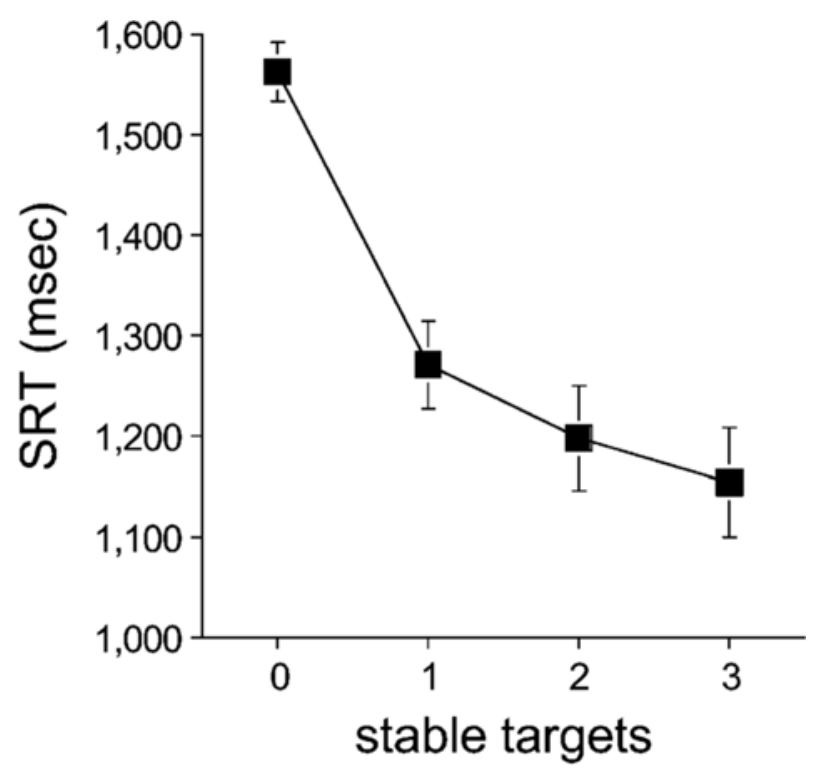

Figure 7. Main effect of target stability. Error bars indicate standard errors of the means. SRT, serial reaction time.

sible. The option to plan two or three steps ahead provides a small additional advantage. One interpretation is that observers rarely plan more than one step ahead, even when the information is available. Trials on which observers plan two or three steps ahead occur often enough to influence the overall SRT, but not nearly as often as trials on which only the next step is plotted in advance. Contrast this with the retrospective memory effect, which seems to keep track of the previous three targets. We can think of this in terms of the observers' temporal focus of attention, which extends beyond the current moment, but further into the past than into the future.

\section{Future Directions}

The main goal of the present article was to explore the influence of past and future actions on responses to a current target. However, more generally, we hope that the present work has demonstrated the potential that this simple multitarget localization task has as a tool for examining both the temporal and the spatial contexts of search and action.

For example, in the present work, we used an arbitrary target sequence of 4 items. What happens when the sequence is extended to 8,10 , or 12 items? Can observers effectively ignore any number of old targets, or if there is a limit, which seems much more likely, what are the capacity limits? Similarly, we used simple, highly familiar target sequences (i.e., alphabetic letters). Does our ability to tag or plan ahead interact with the "spatial" complexity of the sequence? What would happen if observers had to memorize an arbitrary sequence of letters or digits on each trial (e.g., X, P, A, H)? What would happen if the quality of the target items was severely degraded? Would an increase in task difficulty (e.g., perceptual degradation of the target items) remove either prospective or retrospective effects?
We might also want to decompose the search- and the response-related aspects of performance on this task. The search literature typically attributes increases in RT with set size as evidence for difficulty in finding the target (for a review, see Wolfe, 1998a). Data from localization tasks (Meegan \& Tipper, 1998; Pratt \& Abrams, 1994; Tipper et al., 1992) indicate that the presence of distractors increases RT even when targets and distractors are easily distinguished, although there has been little investigation of set size effects. The present task prompts us to ask whether effects attributed to response competition can be explained by search difficulty, or vice versa. The design of the MILO task is particularly amenable to asking these kinds of questions.

More generally, we believe that the introduction of target sequences and direct responses has important theoretical and practical implications. In terms of theory, the MILO task represents important steps forward in terms of both conceptual coverage (temporal as well as spatial domains) and behavioral relevance (selection for action). Of course, on a practical level, these changes may make it difficult to make direct comparisons between findings from the two types of tasks. Rather than treating the MILO task as simply a special case of visual search, we believe that it will be necessary to reexamine established findings from the search literature in the context of this new paradigm. For example, do the same stimuli that allow efficient visual search afford rapid direct selection? Can observers benefit from contextual cuing (Chun \& Jiang, 1998)? Does attentional capture affect multiple responses (Theeuwes \& Godijn, 2002; Yantis, 1993)?

\section{Conclusions}

Our extensive knowledge of the effects of spatial context on visual search are beginning to be complemented by an understanding of the effects of temporal context (Klein \& MacInnes, 1999; Rensink, 2000; Rensink et al., 2000; Wolfe et al., 2000). The MILO task we have introduced here has added to our knowledge of temporal influences by demonstrating that (1) old target items can be efficiently tagged and/or ignored and (2) given a stable item array, observers plan ahead at least as far as the next item in the sequence. Finally, we hope that this new task will prove to be a useful tool for us to ask a wider range of questions about the influence of spatial and temporal contexts in visual search.

\section{REFERENCES}

Adam, J. J., Nieuwenstein, J. H., Huys, R., PaAs, F. G., Kingma, H., Willems, P., \& Werry, M. (2000). Control of rapid aimed hand movements: The one-target advantage. Journal of Experimental Psychology: Human Perception \& Performance, 26, 295-312.

Briand, K. A., LARrison, A. L., \& SEREnO, A. B. (2000). Inhibition of return in manual and saccadic response systems. Perception \& Psychophysics, 62, 1512-1524.

CHun, M. M., \& JiAng, Y. (1998). Contextual cueing: Implicit learning and memory of visual context guides spatial attention. Cognitive Psychology, 36, 28-71.

Donk, M., \& TheEUWES, J. (2001). Visual marking beside the mark: Prioritizing selection by abrupt onsets. Perception \& Psychophysics, 63, 891-900. 
DunCAN, J., \& Humphreys, G. (1989). Visual search and stimulus similarity. Psychological Review, 96, 433-458.

DunCAN, J., \& HumphreYs, G. (1992). Beyond the search surface: Visual search and attentional engagement. Journal of Experimental Psychology: Human Perception \& Performance, 18, 578-588.

Eckstein, M. P., Thomas, J. P., PAlmer, J., \& Shimozaki, S. S. (2000). A signal detection model predicts the effects of set size on visual search accuracy for feature, conjunction, triple conjunction, and disjunction displays. Perception \& Psychophysics, 62, 425-451.

Fischer, M. H., PrATt, J., \& NegGERS, S. F. W. (2003). Inhibition of return and manual pointing movements. Perception \& Psychophysics, 65, 379-387.

Fischer, M. H., Rosenbaum, D. A., \& Vaughan, J. (1997). Speed and sequential effects in reaching. Journal of Experimental Psychology: Human Perception \& Performance, 23, 404-428.

GIBSON, B. S., \& JIANG, Y. (2001). Visual marking and the perception of salience in visual search. Perception \& Psychophysics, 63, 59-73.

Gibson, B. S., Li, L., Skow, E., Salvagni, K., \& Cooke, L. (2000). Memory-based tagging of targets during visual search for one versus two identical targets. Psychological Science, 11, 324-328.

GILCHRIST, I. D., \& HARVEY, M. (2000). Refixation frequency and memory mechanisms in visual search. Current Biology, 10, 1209-1212.

GILCHRIST, I. D., NORTH, A., \& HOOD, B. (2001). Is visual search really like foraging? Perception, 30, 1459-1464.

Hillstrom, A. P. (2000). Repetition effects in visual search. Perception \& Psychophysics, 62, 800-817.

Hollingworth, A., Williams, C. C., \& Henderson, J. M. (2001). To see and remember: Visually specific information is retained in memory from previously attended objects in natural scenes. Psychonomic Bulletin \& Review, 8, 761-768.

Horowitz, T. S., \& Wolfe, J. M. (1998). Visual search has no memory. Nature, 394, 575-577.

Horowitz, T. S., \& Wolfe, J. M. (2001). Search for multiple targets: Remember the targets, forget the search. Perception \& Psychophysics, 63, 272-285.

Horowitz, T. S., \& Wolfe, J. M. (2003). Memory for rejected distractors in visual search? Visual Cognition, 10, 257-298.

Howard, L. A., LuPiáñE Z, J., \& TipPER, S. P. (1999). Inhibition of return in a selective reaching task: An investigation of reference frames. Journal of General Psychology, 126, 421-442.

Keulen, R. F., AdAm, J. J., Fischer, M. H., Kuipers, H., \& Jolles, J. (2002). Selective reaching: Evidence for multiple frames of reference. Journal of Experimental Psychology: Human Perception \& Performance, 28, 515-526.

KLEIN, R. [M.] (1988). Inhibitory tagging system facilitates visual search. Nature, 334, 430-431.

KLEIN, R. M., \& MACINNES, W. J. (1999). Inhibition of return is a foraging facilitator in visual search. Psychological Science, 10, 346-352.

KLEIN, R. M., \& TAYLOR, T. L. (1994). Categories of cognitive inhibition with reference to attention. In D. Dagenbach \& T. H. Carr (Eds.), Inhibitory processes in attention, memory, and language (pp. 113150). San Diego: Academic Press.

KRISTJÁnsSON, A. (2000). In search of remembrance: Evidence for memory in visual search. Psychological Science, 11, 328-332.

KRUEGER, L. E. (1984). The category effect in visual search depends on physical rather than conceptual differences. Perception \& Psychophysics, 35, 558-564.

Maljkovic, V., \& Nakayama, K. (1994). Priming of pop-out: I. Role of features. Memory \& Cognition, 22, 657-672.

Maljkovic, V., \& Nakayama, K. (1996). Priming of pop-out: II. Role of position. Perception \& Psychophysics, 58, 977-991.

MCAulifFe, J., PRATT, J., \& O'DONnEll, C. (2001). Examining locationbased and object-based components of inhibition of return in static displays. Perception \& Psychophysics, 63, 1072-1082.

MCELREe, B., \& CARRASCO, M. (1999). The temporal dynamics of visual search: Evidence for parallel processing in feature and conjunction searches. Journal of Experimental Psychology: Human Perception \& Performance, 25, 1517-1539.

MeEgan, D. V., \& TiPPER, S. P. (1998). Reaching into cluttered visual environments: Spatial and temporal influences of distracting objects. Quarterly Journal of Experimental Psychology, 51A, 225-249.
MeEgAn,D. V., \& TIPPER, S. P. (1999). Visual search and target-directed action. Journal of Experimental Psychology: Human Perception \& Performance, 25, 1347-1362.

Moore, C. M., Egeth, H., Berglan, L. R., \& Luck, S. J. (1996). Are attentional dwell times inconsistent with serial visual search? Psychonomic Bulletin \& Review, 3, 360-365.

MülleR, H., \& VON MÜHLEnEN, A. (2000). Probing distractor inhibition in visual search: Inhibition of return. Journal of Experimental Psychology: Human Perception \& Performance, 26, 1591-1605.

OH, S.-H., \& KIM, M. S. (2002, July). Visual search efficiency is affected by spatial working memory. Paper presented at the Second Asian Conference on Vision, Gyeongju, Korea.

OJANPÄÄ, H., \& NÄSÄNEN, R. (2002). Visual search and simultaneous short-term memory task [Abstract]. Perception, 31 (Suppl.), 174b.

Olivers, C. N. L., \& Humphreys, G. W. (2000). Visual marking is affected by the attentional blink. Perception, 29 (Suppl.), 62-63.

Olivers, C. N. L., Humphreys, G. W., Heinke, D., \& CoOper, A. C. G. (2002). Prioritization in visual search: Visual marking is not dependent on a mnemonic search. Perception \& Psychophysics, 64, 540560 .

Olson, I. R., \& Chun, M. M. (2001). Temporal contextual cuing of visual attention. Journal of Experimental Psychology: Learning, Memory, \& Cognition, 27, 1299-1313.

PAlmer, J. (1995). Attention in visual search: Distinguishing four causes of a set-size effect. Current Directions in Psychological Science, 4, 118-123.

PALMER, J., \& MCLEAN, J. (1995, August). Imperfect, unlimited-capacity, parallel search yields large set-size effects. Paper presented at the annual meeting of the Society of Mathematical Psychology, Irvine, CA.

Pelli, D. G., \& Zhang, L. (1991). Accurate control of contrast on microcomputer displays. Vision Research, 31, 1337-1350.

Peterson, M. S., Kramer, A. F., Wang, R. F., Irwin, D. E., \& MCCARLEY, J. S. (2001). Visual search has memory. Psychological Science, 12, 287-292.

Posner, M. I., \& Cohen, Y. (1984). Components of attention. In H. Bouma \& D. G. Bouwhuis (Eds.), Attention and performance $X$ (pp. 55-66). Hillsdale, NJ: Erlbaum.

Posner, M. I., RAfal, R. D., ChOATE, L. S., \& VAughan, J. (1985). Inhibition of return: Neural basis and function. Cognitive Neuropsychology, 2, 211-228.

PrATt, J., \& ABRAms, R. A. (1994). Action-centered inhibition: Effects of distractors on movement planning and execution. Human Movement Science, 13, 245-254.

REITAN, R. M. (1958). The validity of the trial making test as an indicator of organic brain damage. Perceptual \& Motor Skills, 9, 127-130.

RENSINK, R. A. (1990). Toolbox-based routines for Macintosh timing and display. Behavior Research Methods, Instruments, \& Computers, 22, 105-117.

RENSINK, R. A. (2000). Seeing, sensing, and scrutinizing. Vision Research, 40, 1469-1487.

Rensink, R. A., O'Regan, J. K., \& Clark, J. J. (2000). On the failure to detect changes in scenes across brief interruptions. Visual Cognition, 7, 127-145.

RENSINK, R. A., \& ENNS, J. T. (1995). Preemption effects in visual search: Evidence for low-level grouping. Psychological Review, 102, 101-130.

Ricker, K. L., Elliott, D., Lyons, J., Gauldie, D., ChuA, R., \& BYBLOW, W. (1999). The utilization of visual information in the control of rapid sequential aiming movements. Acta Psychologica, 103, 103123.

Ro, T., \& RAFAL, R. D. (1999). Components of reflexive visual orienting to moving objects. Perception \& Psychophysics, 61, 826-836.

SCHNEIDER, W., \& SHIFFrIN, R. M. (1977). Controlled and automatic human information processing: I. Detection, search, and attention. Psychological Review, 84, 1-66.

SHORE, D. I., \& KLEIN, R. M. (2000). On the manifestations of memory in visual search. Spatial Vision, 14, 59-75.

SNyder, J. J., \& Kingstone, A. (2000). Inhibition of return and visual search: How many separate loci are inhibited? Perception \& Psychophysics, 62, 452-458.

STEINMAN, S. B., \& NAWROT, M. (1992). Real-time color-frame anima- 
tion for visual psychophysics on the Macintosh computer. Behavior Research Methods, Instruments, \& Computers, 24, 439-452.

TAKEDA, Y., \& YAGI, A. (2000). Inhibitory tagging in visual search can be found if search stimuli remain visible. Perception \& Psychophysics, 62, 927-934.

TANAKA, Y., \& SHIMOJO, S. (1996). Location versus feature: Reaction time reveals dissociation between two visual functions. Vision Research, 36, 2125-2140

TheE UWES, J., \& GodiJn, R. (2002). Irrelevant singletons capture attention: Evidence from inhibition of return. Perception \& Psychophysics, 64, 764-770.

Theeuwes, J., Kramer, A. F., \& Atchley, P. (1998). Visual marking of old objects. Psychonomic Bulletin \& Review, 5, 130-134.

TIPPER, S. P., JORDAN, H., \& WEAVER, B. (1999). Scene-based and objectcentered inhibition of return: Evidence for dual orienting mechanisms. Perception \& Psychophysics, 61, 50-60.

TipPeR, S. P., Lortie, C., \& BAYLis, G. C. (1992). Selective reaching: Evidence for action-centered attention. Journal of Experimental Psychology: Human Perception \& Performance, 18, 891-905.

Tipper, S. P., MeEgan, D. V., \& Howard, L. A. (2002). Action-centred negative priming: Evidence for reactive inhibition. Visual Cognition, 9, 591-614.

TipPer, S. P., WeaVer, B., Jerreat, L. M., \& Burak, A. L. (1994). Objectbased and environment-based inhibition of return of visual attention. Journal of Experimental Psychology: Human Perception \& Performance, 20, 478-499.

Treisman, A. (1993). The perception of features and objects. In A. D. Baddeley \& L. Weiskrantz (Eds.), Attention: Selection, awareness, and control. A tribute to Donald Broadbent (pp. 5-35). Oxford: Oxford University Press, Clarendon Press.

TREISMAN, A., \& HAYES, A. (1998). Location and feature specificity in priming and automatized search. Unpublished manuscript.

Treisman, A., \& SATO, S. (1990). Conjunction search revisited. Journal of Experimental Psychology: Human Perception \& Performance, 16, 459-478.

VAN Selst, M., \& JolicÄUR, P. (1997). Decision and response in dualtask interference. Cognitive Psychology, 33, 266-307.

WATSON, D. G. (2001). Visual marking in moving displays: Featurebased inhibition is not necessary. Perception \& Psychophysics, 63, 7484.

Watson, D. G., \& Humphreys, G. W. (1997). Visual marking: Prioritizing selection for new objects by top-down attentional inhibition of old objects. Psychological Review, 104, 90-122.

WATSON, D. G., \& HuMPhre Ys, G. W. (2000). Visual marking: Evidence for inhibition using a probe-dot detection paradigm. Perception \& Psychophysics, 62, 471-481.

Wolfe, J. M. (1994). Guided Search 2.0: A revised model of visual search. Psychonomic Bulletin \& Review, 1, 202-238.
Wolfe, J. M. (1998a). Visual search. In H. Pashler (Ed.), Attention (pp. 13-73). Hove, U.K.: Psychology Press.

WoLfe, J. M. (1998b). What can 1 million trials tell us about visual search? Psychological Science, 9, 33-39.

Wolfe, J. M. (1999). Inattentional amnesia. In V. Coltheart (Ed.), Fleeting memories: Cognition of brief visual stimuli (pp. 71-94). Cambridge, MA: MIT Press.

Wolfe, J. M., Klempen, N., \& DAhlen, K. (2000). Postattentive vision. Journal of Experimental Psychology: Human Perception \& Performance, 26, 693-716.

WoOdman, G. F., Voge L, E. K., \& LUCK, S. J. (2001). Visual search remains efficient when working memory is full. Psychological Science, 12, 219-224.

WRIGHT, R. D., \& RICHARD, C. M. (1996). Inhibition of return at multiple locations in visual space. Canadian Journal of Experimental Psychology, 50, 324-327.

YANTIS, S. (1993). Stimulus-driven attentional capture and attentional control settings. Journal of Experimental Psychology: Human Perception \& Performance, 19, 676-681.

ZENGER, B., \& FAHLE, M. (1997). Missed targets are more frequent than false alarms: A model for error rates in visual search. Journal of Experimental Psychology: Human Perception \& Performance, 23, 1783 1791.

\section{NOTES}

1. Since completing these experiments, our attention has been drawn to a well-established neuropsychological task, the trail-making test (e.g., Reitan, 1958). The paper-and-pencil task also involves observers' searching through an array of targets and making sequential responses. However, in the trail-making task, the only available measurements of interest are usually total errors and/or total time on task. Since the application domain is very different and the paper-and-pencil implementation does not permit the manipulations we are exploring, we have not draw any parallels with this literature.

2. Although a fixation cross was always presented in the center of the screen, in the present series of experiments, the observers were never required to maintain fixation during any part of a trial. The fixation cross was included in the display to help localize the items during the cuing part of the trial and also to provide an identical display layout if we ever do decide to run fixation conditions.

3. Note that whether the search and the response stages were strictly serial or whether there was some overlap is not important for our purposes here.

(Manuscript received November 19, 2002; revision accepted for publication May 20, 2003.) 